\title{
Primärärztliche Strategien und Zusammenarbeit während der ersten Phase der COVID-19-Pandemie in Baden-Württemberg, Deutschland
}

\section{Primary Care Strategies and Cooperation During the First Phase of the COVID-19 Pandemic in Baden-Wuerttemberg, Germany}

\author{
Autoren

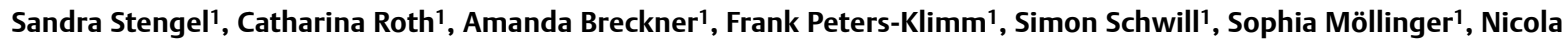 \\ Buhlinger-Göpfarth ${ }^{2}$, Joachim Szecsenyi ${ }^{1}$, Michel Wensing ${ }^{1}$
}

Institute

1 Abteilung Allgemeinmedizin und Versorgungsforschung, UniversitätsKlinikum Heidelberg,Heidelberg, Deutschland

275181 Pforzheim, Gemeinschaftspraxis Dr. Nicola Buhlinger-Göpfarth \& Dr. Eleonore Fritz, Pforzheim, Deutschland

\section{Schlüsselwörter}

COVID-19, Pandemie, ambulante Versorgung, primärärztliche Versorgung, Deutschland

Key words

COVID-19, pandemic, outpatient care, primary care, Germany

online publiziert $\quad 19.03 .2021$

\section{Bibliografie}

Gesundheitswesen 2021; 83: 250-257

DOI 10.1055/a-1397-7527

ISSN $0941-3790$

(C) 2021. Thieme. All rights reserved.

Georg Thieme Verlag KG, Rüdigerstraße 14,

70469 Stuttgart, Germany

\section{Korrespondenzadresse}

Sandra Stengel

Abteilung Allgemeinmedizin und Versorgungsforschung

UniversitätsKlinikum Heidelberg

Im Neuenheimer Feld 130.3

69120 Heidelberg

Deutschland

sandra.stengel@med.uni-heidelberg.de

\section{ZUSAMMENFASSUNG}

Ziel der Studie Das Ziel der Arbeit war eine deskriptive frühzeitige Momentaufnahme von Einleitung und Umsetzung ambulanter Strategien im primärärztlichen Setting zur Bewältigung der frühen Phase der COVID-19- Pandemie in BadenWürttemberg (Deutschland).
Methodik Im Juni 2020 erhielten alle 271 Corona-Anlaufstellen unter Trägerschaft der Kassenärztlichen Vereinigung (16 Abstrichstellen, 204 Corona-Schwerpunktpraxen, 51 Zentrale Fieberambulanzen) sowie eine zufällig generierte Stichprobe von 400 Hausarztpraxen aus Baden-Württemberg einen papierbasierten Fragebogen. Die Daten wurden anonym erhoben und deskriptiv ausgewertet.

Ergebnisse Insgesamt nahmen $n=63(15,8 \%)$ Hausarztpraxen und $\mathrm{n}=92(33,9 \%)$ Corona-Anlaufstellen teil. 78,7\% der Hausarztpraxen nutzten Corona-Anlaufstellen $(n=48) .92,1 \%$ hatten eine verpflichtende telefonische Anmeldung für PatientInnen mit (vermuteter) COVID-19-Erkrankung $(n=58)$ implementiert. $81 \%$ boten bei leichtem Verlauf eine rein telefonische oder videokonsultatorische Versorgung an ( $n=51)$. Parallel dazu wurden die neuen ambulanten Corona-Anlaufstellen vorwiegend unter hausärztlicher Leitung ( $n=76,82,6 \%$ ) in Zusammenarbeit mit weiteren Akteuren, in fast der Hälfte der Fälle bereits im März aufgebaut ( $n=42,48,3 \%$ ). Die am häufigsten genannten Anmeldepfade waren gesteuert und konnten vorwiegend über HausärztInnen ( $n=88,95,7 \%)$ und Gesundheitsamt ( $n=74,80,4 \%$ ), aber auch durch weitere Akteure erfolgen. In 92,4\% ( $n=85)$ konnte eine telefonische Anmeldung erfolgen. Die mündliche Rückmeldung an die PatientInnen ( $n=65,77,4 \%$ ) war der am häufigsten genannte Rückmeldeweg. In weniger als der Hälfte der Corona-Anlaufstellen lagen standardisierte Anmelde-, Dokumentations- und Rückmeldebögen vor. Die Einschätzung der zukünftigen Versorgungsstrukturen von PatientInnen mit (vermuteter) COVID19-Erkrankung waren heterogen.

Schlussfolgerungen In einem gemeinsamen Kraftakt, mit Improvisation und Zusammenarbeit gelang eine rasche Implementierung von Maßnahmen zur Patientenversorgung während der Anfangsphase der Pandemie im primärärztlichen Setting. Aus den Ergebnissen können Impulse für die primärärztliche Versorgung in einer Pandemie abgeleitet werden.

\section{ABSTRACT}

Objectives The aim of this study was to give an early snapshot of primary care strategies that were implemented to cope with 
the early period of the COVID-19 pandemic in Baden-Wuerttemberg (Germany).

Methods In June 2020, all 271 outpatient SARS-CoV-2 contact points, established by the National Association of Statutory Health Insurance Physicians (16 centers for testing, 204 specialized family practices, 51 Outpatients Corona Centers), and a randomly generated sample of 400 primary care practices of Baden-Wuerttemberg were invited to take part in a paper-based questionnaire. The data were gathered anonymously and analysed descriptively.

Results Out of those invited, $\mathrm{n}=63(15.8 \%)$ primary care practices and $\mathrm{n}=92$ (33.9\%) SARS-CoV-2 contact points participated; $78.7 \%$ of the primary care practices cooperated with SARSCoV-2 contact points $(n=48)$. In all, $92.1 \%$ had implemented a compulsory registration by phone for patients with (suspected) COVID-19 $(n=58)$ and $81 \%$ offered consultation exclusively by phone or video in case of a mild courses $(n=51)$. The new outpatient SARS-CoV-2 contact points were established in collabo- ration with several stakeholders, mainly led by primary care physicians ( $n=76,82.6 \%$ ) and almost $50 \%$ of these were established in March 2020 ( $n=42,48.3 \%)$. The most commonly reported method of registration was regulated mainly by primary care practices $(n=88,95.7 \%)$ and public health departments ( $n=74,80.4 \%)$. In $92.4 \%(n=85)$ of cases, it was possible to register by phone. The consultation response was most commonly given in the form of oral information to the patient $(n=65$, $77.4 \%$ ). Less then $50 \%$ of the SARS-CoV-2 contact points used standardized sheets for registration, documentation and consultation. The assessment of future primary care structures for (suspected) COVID-19 patients were heterogeneous.

Conclusions Effort, improvisation and collaboration were required for a successful and rapid implementation of measures for primary care during the initial period of the COVID-19 pandemic. Impulses for ongoing development of primary care strategies during a pandemic can be derived out of these results.

\section{Einleitung}

Der erste SARS-CoV-2-Nachweis erfolgte in Deutschland am 27. Januar 2020 in Bayern [1]. Aktuell sind 2205171 SARS-CoV-2-Infektionen mit 56546 Todesfällen in Deutschland und 291818 SARS-CoV-2-Infektionen mit 7013 Todesfällen in Baden-Württemberg beschrieben (Stand 30.01.2021) [2]. An der Bewältigung der COVID-19-Pandemie sind im deutschen Gesundheitswesen maßgeblich der öffentliche Gesundheitsdienst, der stationäre und ambulante Sektor neben weiteren Akteuren beteiligt [3]. In Deutschland soll dabei laut Pandemieplänen zur Sicherstellung der notwendigen stationären Behandlungsressourcen für schwer erkrankte Fälle die Patientenversorgung möglichst ambulant erfolgen und stationär versorgte Patientlnnen frühzeitig entlassen werden [35]. Die ambulante Versorgung obliegt im Rahmen des Sicherstellungsauftrags nach $§ 72$ SGB V den Kassenärztlichen Vereinigungen [6]. Konkrete Handlungsanweisungen für den ambulanten Sektor sind auf Empfehlungen für das Risikomanagement in Arztpraxen im Falle einer Influenzapandemie beschränkt [7]. Am 12.03.2020 wurden vom Robert-Koch-Institut erstmalig Maßnahmen zur getrennten Versorgung von (vermuteten) COVID-19- und Nicht-COVID-19-PatientInnen im ambulanten und prästationären Bereich veröffentlicht [8]. Hier werden verschiedene Testmöglichkeiten, COVID-Ambulanzen und Empfehlungen mit räumlicher und zeitlicher Trennung für Praxen aufgezählt.

Laut Kassenärztlicher Bundesvereinigung wurde in Deutschland der überwiegende Anteil der COVID-19-PatientInnen bislang ambulant versorgt [9]. Weltweit ist das komplexe Einwirken der COVID-19-Pandemie auf den primärärztlichen Bereich beschrieben [10]. In der ersten Phase der COVID-19-Pandemie kam es in Deutschland in der sehr dynamischen Ausbreitungssituation zur Notwendigkeit der ad-hoc-Implementierung der o.g. (teils erst nachfolgend) empfohlenen Maßnahmen. Neben Umstrukturierungen in der hausärztlichen Versorgung kam es dabei in Baden-Württemberg zum Aufbau neuer ambulanter Versorgungsformen unter Trägerschaft der Kassenärztlichen Vereinigung Baden-Württem- berg (KVBW), im Folgenden als Corona-Anlaufstellen bezeichnet: Abstrichstellen, Corona-Schwerpunktpraxen und Zentrale Fieberambulanzen [11]. In Abstrichstellen besteht die Möglichkeit einer Testung auf SARS-CoV-2. Corona-Schwerpunktpraxen (Betriebsstättennummer und Organisation durch eine bestehende Hausarztpraxis) und zentrale Fieberambulanzen (eigene Betriebsstättennummer) bieten die Möglichkeit von Diagnostik und Untersuchung bei PatientInnen mit Symptomen von COVID-19.

Das Ziel der Arbeit war deskriptiv in einer frühzeitigen Momentaufnahme zu erfassen, welche ambulanten Strategien im primärärztlichen Setting zur Bewältigung der frühen Phase der COVID19-Pandemie in Baden-Württemberg eingeleitet wurden, wie diese umgesetzt wurden und wie die Zusammenarbeit an der Schnittstelle der Corona-Anlaufstellen gestaltet war.

\section{Methodik}

\section{Studiendesign und -setting}

Die deskriptive Beobachtungsstudie wurde in der Frühphase der COVID-19-Pandemie geplant und nach Erhalt eines positiven Ethikvotums der Ethikkommission der Medizinischen Fakultät Heidelberg (S-418/2020) sowie Registrierung im Deutschen Register für klinische Studien (DRKS00022224) durchgeführt. Der Bericht orientiert sich an den STROBE-Kriterien für Beobachtungsstudien [12]. Es erfolgte eine anonymisierte, schriftliche Befragung von leitendem Personal von Corona-Anlaufstellen und Hausarztpraxen mittels eines papierbasierten Fragebogens.

\section{Studienpopulation und Rekrutierung}

Es wurden alle Corona-Anlaufstellen und hausärztlich tätigen ÄrztInnen mit Kassenarztzulassung in Baden-Württemberg über die Webseite der KVBW identifiziert. Der Stand am 15.06.2020 betrug 16 Abstrichstellen, 51 Zentrale Fieberambulanzen, 204 CoronaSchwerpunktpraxen und 8135 HausärztInnen. Zur Verminderung 
eines Bias wurden alle Hausarztpraxen ausgeschlossen, die Corona-Schwerpunktpraxen waren. Hinsichtlich der Corona-Anlaufstellen erfolgte eine Vollerhebung. In der Gruppe der Hausarztpraxen wurde eine zufällige Stichprobe von $n=400$ mittels Zufallszahlgenerator gezogen unter Berücksichtigung aller Land- und Stadtkreise analog ihrer Verteilung nach Einwohnerzahl. Am 15. Juni 2020 wurden alle Leiterlnnen der 271 Corona-Anlaufstellen sowie die Stichprobe von $n=400$ Hausarztpraxen aus Baden-Württemberg postalisch eingeladen an der schriftlichen Befragung teilzunehmen. Am 20. Juli 2020 wurde die Datenerhebung beendet. In der Gruppe der Hausarztpraxen wurden nach der Rücksendung Personen ausgeschlossen, die auch in einer Corona-Anlaufstelle tätig waren, um das Risiko eines Bias zu vermindern.

\section{Variablen und Datenauswertung}

Die Fragebögen für Corona-Anlaufstellen und Hausarztpraxen wurden in einem interdisziplinären Team auf Grundlage von 6 Orientierungsinterviews mit HausärztInnen entwickelt, die Pilotierung erfolgte in einem Testlauf mit Hausärztlnnen aus dem Studienteam. Die Fragebögen enthielten Fragen zu Soziodemografie, Struktur/ Organisation, Patientenkontakten/Kapazität, Anamnese/Diagnostik/Dokumentation/Versorgung/Rückmeldung/Schnittstellen, Einstellung/Haltung, Wissensquellen/Wissensstand, Maßnahmen zur Eindämmung der SARS-CoV-2-Pandemie, Inanspruchnahme, weitere Versorgung von PatientInnen mit (vermuteter) COVID-19-Erkrankung sowie die Option einer Freitexteingabe. Die Häufigkeiten der nominalen bzw. ordinalen Antwortoptionen zu ausgewählten Fragen wurden mittels der Statistiksoftware IBM SPSS Version 25.0 deskriptiv berechnet. Die Häufigkeiten wurden bezogen auf die Anzahl der verwertbaren Rückmeldungen. Bei den ordinalen Antwortoptionen „trifft ganz zu“ - „trifft weitgehend zu“ - „trifft teils zu, teils nicht zu“ - „trifft weitgehend nicht zu“- „trifft überhaupt nicht zu“ - „kann ich nicht beurteilen“ wurden die erste und zweite Antwortoption als „trifft zu“ und die vierte und fünfte. als „trifft nicht zu“ wegen der geringen Anzahl an Nennungen zusammengefasst. Ein statistischer Vergleich zwischen den Gruppen der Corona-Anlaufstellen wurde aufgrund der geringen Fallzahlen in der Gruppe der Fieberambulanzen nicht durchgeführt.

\section{Ergebnisse}

\section{Studienpopulation}

Von den 271 angeschriebenen Corona-Anlaufstellen füllten $\mathrm{n}=92$ $(33,9 \%)$ den Fragebogen aus. Die Rücklaufquote betrug in der Gruppe der Fieberambulanzen n=16 (31,4\%), der Corona-Schwerpunktpraxen $n=74$ (36,3\%) und der Abstrichstellen $n=2$ (12,5\%). Die Rücklaufquote in der Gruppe der Hausarztpraxen betrug $n=79$ $(19,8 \%)$. Hiervon wurden $n=16$ ausgeschlossen, weil sie eine Tätigkeit in einer Corona-Anlaufstelle angaben. Nach Ausschluss konnten $n=63$ (15,8\%) ausgewertet werden. In $>$ Tab. 1 wird die Studienpopulation beschrieben. Die überwiegende Mehrheit der LeiterInnen der Corona-Anlaufstellen waren Hausärztlnnen ( $n=76$, $82,6 \%$ ). Die Verteilung der Corona-Anlaufstellen und Hausarztpraxen bezogen auf den Standort und das Alter war ähnlich.

\section{Beschreibung des primärärztlichen Settings}

Die Ergebnisse zu Inbetriebnahme, zusätzlichen Versorgungsangeboten und Öffnungszeiten finden sich in $>$ Tab. 2. Die Inbetriebnahme der Corona-Anlaufstellen erfolgte überwiegend im März 2020 ( $n=42,48,3 \%)$ und April 2020 ( $n=34,39,1 \%)$.

Die hausärztlichen Ergebnisse zur Nutzung von Corona-Anlaufstellen, Einschätzung der Inanspruchnahme, Implementierung von verpflichtender telefonischer Anmeldung und ausschließlicher Versorgung per Telefon bzw. Video finden sich ebenfalls in $>$ Tab. 2. Der überwiegende Anteil der Hausarztpraxen ( $n=48,78,7 \%$ ) nutzte Corona-Anlaufstellen. Die Anzahl der Hausarztpraxen mit wahrgenommener reduzierter Inanspruchnahme von Nicht-COVID-PatientInnen reduzierte sich von einem Maximum mit n= $59(93,7 \%)$ in der Osterzeit auf $n=33(52,4 \%)$ zum Befragungszeitpunkt Mitte - Ende Juni 2020.

\section{Gegenüberstellung von Corona-Schwerpunktpraxen und zentralen Fieberambulanzen im Aufbau}

Die Ergebnisse finden sich in > Tab. 3. Corona-Schwerpunktpraxen wurden vorwiegend in eigenen Praxisräumen eingerichtet $(n=57$, $78,1 \%$ ), Fieberambulanzen ( $n=15,93,8 \%$ ) hingegen überwiegend in anderen Räumen (fremde Praxisräume, Klinikräume, Hallen, Con-

- Tab. 1 Charakterisierung der Studienpopulation.

\begin{tabular}{|c|c|c|}
\hline Merkmale & $\begin{array}{l}\text { Corona- } \\
\text { Anlaufstel- } \\
\text { len } \mathbf{n = 9 2}\end{array}$ & $\begin{array}{l}\text { Hausarzt- } \\
\text { praxen } \\
n=63\end{array}$ \\
\hline \multicolumn{3}{|l|}{ Altersgruppe, n (\%) ${ }^{a}$} \\
\hline unter 30 Jahre & $1(1,1)$ & 0 \\
\hline zwischen 30 und 40 Jahren & $13(14,1)$ & $7(11,1)$ \\
\hline zwischen 41 und 50 Jahren & $27(29,3)$ & $16(25,4)$ \\
\hline zwischen 51 und 60 Jahren & $39(42.4)$ & $26(41,3)$ \\
\hline über 60 Jahre & $11(12,0)$ & $14(22,2)$ \\
\hline keine Angabe & $1(1,1)$ & 0 \\
\hline \multicolumn{3}{|l|}{ Standort, n (\%) ${ }^{a}$} \\
\hline Stadtzentrum & $44(47,8)$ & $36(57,1)$ \\
\hline Stadt-Umkreis (20 km) & $31(33,7)$ & $17(27,0)$ \\
\hline Ländliches Gebiet (Stadt> 20 km) & $17(18,5)$ & $8(12,7)$ \\
\hline Keine Angabe & 0 & $2(3,2)$ \\
\hline \multicolumn{3}{|l|}{ Geschlecht, n (\%) a } \\
\hline Weiblich & $30(32,6)$ & $32(50,8)$ \\
\hline Männlich & $61(66,3)$ & $31(49,2)$ \\
\hline keine Angabe & $1(1,1)$ & 0 \\
\hline \multicolumn{3}{|l|}{ Berufliche Qualifikation, n (\%) ${ }^{\text {b }}$} \\
\hline Hausärztlnnenc & $76(82,6)$ & \multirow{3}{*}{$\mathrm{n} / \mathrm{a}$} \\
\hline Andere FachärztInnen & $13(14,1)$ & \\
\hline Nichtärztliche Mitarbeitende ${ }^{d}$ & $3(3,3)$ & \\
\hline \multicolumn{3}{|c|}{ Corona-Anlaufstellen - Subtypen, n (\%) ${ }^{a}$} \\
\hline Zentrale Fieberambulanz (ZFA) & $16(17,4)$ & \multirow{3}{*}{$\mathrm{n} / \mathrm{a}$} \\
\hline Corona-Schwerpunktpraxis (CSP) & $74(80,4)$ & \\
\hline Abstrichstelle & $2(2,2)$ & \\
\hline \multicolumn{3}{|c|}{$\begin{array}{l}\text { a Einfachantwort. b Mehrfachantworten. c Beinhaltet sowohl } \\
\text { FachärztInnen für Allgemeinmedizin als auch FachärztInnen für } \\
\text { Innere Medizin mit aktuell hausärztlicher Tätigkeit. }{ }^{d} \text { Gesundheits- } \\
\text { und KrankenpflegerInnen, Medizinische Fachangestellte. }\end{array}$} \\
\hline
\end{tabular}


- Tab. 2 Beschreibung des primärärztlichen Settings in der ersten Phase der COVID-19-Pandemie.

\begin{tabular}{|c|c|}
\hline Merkmale (Anzahl verwertbare/mögliche Rückmeldungen) & Corona-Anlaufstellen $\mathbf{n}(\%)$ \\
\hline \multicolumn{2}{|l|}{ Inbetriebnahme der Corona-Anlaufstelle ${ }^{\text {a }}(87 / 92)$} \\
\hline Februar 2020 & $5(5,7)$ \\
\hline März 2020 & $42(48,3)$ \\
\hline April 2020 & $34(39,1)$ \\
\hline Mai 2020 & $5(5,7)$ \\
\hline Juni 2020 & $1(1,1)$ \\
\hline \multicolumn{2}{|l|}{ 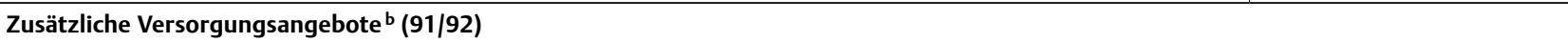 } \\
\hline Mindestens 1 Versorgungsangebot für die Häuslichkeit (Corona-Taxi, Mitbetreuung Pflegeheime, Hausbesuche) & $65(71,4)$ \\
\hline \multicolumn{2}{|l|}{ Öffnungszeiten in Tagen ${ }^{\mathrm{b}}(91 / 92)$} \\
\hline Täglich (Montag bis Freitag) & $82(90,1)$ \\
\hline Zusätzlich samstags & $13(14,3)$ \\
\hline Zusätzlich sonntags & $11(12,1)$ \\
\hline Tageweise (1-2 oder 3-4 Tage/Woche) & $11(12,1)$ \\
\hline Merkmale (Anzahl verwertbare/mögliche Rückmeldungen) & Hausarztpraxen $\mathbf{n}(\%)$ \\
\hline \multicolumn{2}{|l|}{ Zuweisung von PAT_COV neben der Versorgung per Telefon oder Videokonsultation an b,d (61/63) } \\
\hline Abstrichstellen & $24(39,3)$ \\
\hline Zentrale Fieberambulanz & $29(47,5)$ \\
\hline Corona-Schwerpunktpraxen & $17(27,9)$ \\
\hline Klinik & $18(29,5)$ \\
\hline Keine & $13(21,3)$ \\
\hline \multicolumn{2}{|l|}{ Erniedrigte Inanspruchnahme durch PAT_NICHT_COV zu Beginn der COVID-19 Pandemie a,c,d (63/63) } \\
\hline Trifft zu & $52(82,5)$ \\
\hline \multicolumn{2}{|l|}{ Erniedrigte Inanspruchnahme durch PAT_NICHT_COV in der Osterzeit $2020^{a, c}(63 / 63)$} \\
\hline Trifft zu & $59(93,7)$ \\
\hline \multicolumn{2}{|l|}{ Erniedrigte Inanspruchnahme durch PAT_NICHT_COV zum Befragungszeitpunkt (Juni 2020) a,c,d (63/63) } \\
\hline Trifft zu & $33(52,4)$ \\
\hline \multicolumn{2}{|l|}{ Verpflichtende telefonische Anmeldung vor Terminvereinbarung durch PAT_COV a,c,d (63/63) } \\
\hline Trifft zu & $58(92,1)$ \\
\hline \multicolumn{2}{|l|}{ Ausschließlich telefonische oder Videokonsultation-Behandlung von PAT_COV mit leichtem Verlaufa,c,d (63/63) } \\
\hline Trifft zu & $51(81,0)$ \\
\hline
\end{tabular}

tainer, Zelte u. a.). Die beiden Abstrichstellen wurden in Containern organisiert. Sonstige Angaben waren unter anderem eine ehemalige Kaserne, Garagen oder eine umgebaute Wohnung.

Hinsichtlich der Initiative der Einrichtung und inhaltlichen Unterstützung fallen Mehrfachnennungen auf. Besonders häufig wurden bei den Corona-Schwerpunktpraxen die KVBW ( $n=38,51,4 \%$; $n=41,59,4 \%)$ und die Eigeninitiative ( $n=34,45,9 \% ; n=17,24,6 \%)$ genannt. Bei den Fieberambulanzen wurden besonders häufig die Ärzteschaft ( $n=12,75 \% ; n=11,68,8 \%)$, die KVBW ( $n=9,56,3 \%$; $n=10,62,5 \%)$ und das Gesundheitsamt ( $n=9,56,3 \% ; n=5,31,3 \%)$ genannt. Sonstige Organisationen ( $n=13,18,8 \%)$, die beim Aufbau der Corona-Schwerpunktpraxen inhaltlich unterstützt haben, waren das Deutsche Rote Kreuz, die Deutsche Lebensrettungsgesellschaft und die Malteser.

Die Dauer des Aufbaus erfolgte bei einem Großteil der CoronaAnlaufstellen unter 1 Woche $(n=80,88,0 \%)$. Dabei war tendenziell der Aufbau der Corona-Schwerpunktpraxen schneller als bei den Fieberambulanzen. $n=33$ (45,2\%) der Corona-Schwerpunktpraxen und $n=13(81,3 \%)$ der Fieberambulanzen empfanden den Aufbau als aufwendig.

In den Corona-Schwerpunktpraxen überwog mit n= 52 (70,3\%) die vollständig digitale Dokumentation, wohingegen die Mehrheit der Fieberambulanzen ( $n=11,68,8 \%$ ) sowie die beiden Abstrichstellen teilweise digitale und teilweise papier-basierte Dokumentation angaben. Angegebene Gründe hierfür waren Schwierigkeiten mit der IT, dass keine Hard- oder Software zur Verfügung standen oder hygienische Gründe

\section{Zusammenarbeit an der Schnittstelle Corona-Anlaufstelle}

Die Ergebnisse seitens der Corona-Anlaufstellen zu Anmeldung, Rückmeldung, Standardisierung der Anmeldung/Dokumentation/ Rückmeldung und Zufriedenheit mit der Inanspruchnahme finden sich in > Tab. 4. Die am häufigsten genannten Anmeldepfade für PatientInnen in den Corona-Anlaufstellen waren gesteuert und konnte vorwiegend über HausärztInnen ( $n=88$, 95,7\%) und Ge- 
- Tab.3 Gegenüberstellung von Corona-Schwerpunktpraxen und zentralen Fieberambulanzen im Aufbau.

\begin{tabular}{|c|c|c|}
\hline Merkmale (Anzahl verwertbare/mögliche Rückmeldungen) & $\begin{array}{l}\text { Corona-Schwerpunktpraxen } \\
\text { n (\%) }\end{array}$ & $\begin{array}{l}\text { Zentrale Fieberambulanzen } \\
\mathrm{n}(\%)\end{array}$ \\
\hline Räumlichkeiten b & $(73 / 74)$ & $(16 / 16)$ \\
\hline Vorhandene eigene Praxisräume & $57(78,1)$ & $1(6,3)$ \\
\hline Andere (fremde Praxisräume, Klinikräume, Hallen, Container, Zelte, Sonstiges) & $16(21,9)$ & $15(93.8)$ \\
\hline Initiative der Einrichtung $\mathrm{b}$ & $(74 / 74)$ & $(16 / 16)$ \\
\hline Kassenärztliche Vereinigung (KV) & $38(51,4)$ & $9(56,3)$ \\
\hline Gesundheitsamt & $5(6,8)$ & $9(56,3)$ \\
\hline Landratsamt & $3(4,1)$ & $8(50,0)$ \\
\hline Kommune & $3(4,1)$ & $4(25,0)$ \\
\hline Ärzteschaft & $17(23,0)$ & $12(75,0)$ \\
\hline Kliniken & 0 & $6(37,5)$ \\
\hline Eigeninitiative & $34(45,9)$ & $2(12,5)$ \\
\hline Sonstige & $1(1,4)$ & 0 \\
\hline Inhaltliche Unterstützung des Aufbaus b & $(69 / 74)$ & $(16 / 16)$ \\
\hline Kassenärztliche Vereinigung (KV) & $41(59,4)$ & $10(62,5)$ \\
\hline Gesundheitsamt & $11(15,9)$ & $5(31,3)$ \\
\hline Landratsamt & $5(7,2)$ & $9(56,3)$ \\
\hline Kommune & $12(17,4)$ & $4(25,0)$ \\
\hline Ärzteschaft & $13(18,8)$ & $11(68,8)$ \\
\hline Kliniken & 0 & $2(12,5)$ \\
\hline Eigeninitiative & $17(24,6)$ & $2(12,5)$ \\
\hline Sonstige & $13(18,8)$ & $3(18,8)$ \\
\hline Dauer des Aufbaus ${ }^{b}$ & $(73 / 74)$ & $(16 / 16)$ \\
\hline $1 \mathrm{Tag}$ & $18(24,7)$ & 0 \\
\hline 2-3 Tage & $25(34,3)$ & $4(25,0)$ \\
\hline 4-6 Tage & $23(31,5)$ & $8(50,0)$ \\
\hline 1 bis 2 Wochen & $3(4,1)$ & $2(12,5)$ \\
\hline mehr als 2 Wochen & $4(5,5)$ & $2(12,5)$ \\
\hline Der Aufbau der Corona-Anlaufstelle war aufwendig a,c & $(73 / 74)$ & $(16 / 16)$ \\
\hline Trifft zu & $33(45,2)$ & $13(81,3)$ \\
\hline Dokumentation $^{\mathrm{a}}$ & $(74 / 74)$ & $(16 / 16)$ \\
\hline Vollständig digital & $52(70,3)$ & $4(25)$ \\
\hline Teilweise digital, teilweise papier-basiert & $20(27)$ & $11(68,8)$ \\
\hline Vollständig papierbasiert & $2(2,7)$ & $1(6,2)$ \\
\hline
\end{tabular}

sundheitsamt ( $n=74,80,4 \%$ ), aber auch durch weitere Akteure erfolgen. 18,5\% ( $n=17)$ der Corona-Anlaufstellen ermöglichten eine ungesteuerte Behandlung ohne Anmeldung. 27,2\% ( $n=25)$ gewährten auch die ungesteuerte Anmeldung durch PatientInnen selbst. Der am häufigsten genannte Anmeldeweg in den CoronaAnlaufstellen war der telefonische ( $n=85,92,4 \%)$. Der am häufigsten genannte Rückmeldeweg über das Ergebnis der Konsultation war der mündliche an die PatientInnen ( $n=65,77,4 \%$ ). Ebenfalls in - Tab. 4 finden sich die Einschätzungen der Hausarztpraxen über den Informationsstand in Bezug auf die Corona-Anlaufstellen.

\section{Zukunft und Weiterentwicklung}

Die Ergebnisse zur Zukunft und Weiterentwicklung in der Versorgung in Corona-Anlaufstellen und in Hausarztpraxen finden sich in - Tab. 5. Aus Sicht der Corona-Anlaufstellen wurde am häufigsten mit 67,9\% ( $n=53)$ eine Steigerung von Informationsangeboten für HausärztInnen und mit 74,4\% ( $n=58)$ eine Steigerung von Informationsangeboten für PatientInnen als Möglichkeiten zur Steigerung der Inanspruchnahme der eigenen Einrichtungen angegeben. Die Steuerung der ambulanten PatientInnen durch entsprechende Abrechnungsregelungen wurde mit $n=34$ (43,6\%) am wenigsten häufig als Möglichkeit zur Steigerung der Inanspruchnahme angegeben.

\section{Diskussion}

In der vorliegenden Arbeit konnten in einer frühzeitigen Momentaufnahme die ambulanten Strategien und Umsetzungen im primärärztlichen Setting zur Bewältigung der frühen Phase der COVID-19-Pandemie beschrieben werden: Innerhalb kürzester Zeit wurden in den meisten Hausarztpraxen Maßnahmen wie Inan- 
- Tab. 4 Zusammenarbeit an der Schnittstelle Corona-Anlaufstelle.

\begin{tabular}{|c|c|}
\hline Merkmale (Anzahl verwertbare/mögliche Rückmeldungen) & Corona-Anlaufstellen $\mathbf{n}(\%)$ \\
\hline \multicolumn{2}{|c|}{ Die Anmeldung in der Corona-Anlaufstelle kann erfolgen durch ${ }^{\mathrm{b}}$ (92/92) } \\
\hline ohne Anmeldung & $17(18,5)$ \\
\hline Hausarztpraxis & $88(95,7)$ \\
\hline Gesundheitsamt & $74(80,4)$ \\
\hline 116117 & $65(70,7)$ \\
\hline Kliniken & $40(43,5)$ \\
\hline ärztlichen Bereitschaftsdienst & $42(45,7)$ \\
\hline Pflegeheime & $46(50,0)$ \\
\hline PatientInnen telefonisch selbst & $25(27,2)$ \\
\hline \multicolumn{2}{|l|}{ Die Anmeldung erfolgt per ${ }^{b}(92 / 92)$} \\
\hline Telefon & $85(92,4)$ \\
\hline Fax & $37(40,2)$ \\
\hline E-Mail & $31(33,7)$ \\
\hline Sonstige & $10(10,9)$ \\
\hline \multicolumn{2}{|l|}{ Die Rückmeldung erfolgt an/per ${ }^{\mathrm{b}}(84 / 92)$} \\
\hline PatientIn mündlich & $65(77,4)$ \\
\hline PatientIn im Papierformat & $23(27,4)$ \\
\hline Zuweisende Hausarztpraxis per Telefon & $26(31,0)$ \\
\hline Zuweisende Hausarztpraxis per Fax & $47(56,0)$ \\
\hline Zuweisende Hausarztpraxis per Mail & $12(14,3)$ \\
\hline Zuweisende Hausarztpraxis durch Patientln & $14(16,7)$ \\
\hline Sonstige (Kliniken, Gesundheitsamt, keine Rückmeldung) & $17(20,2)$ \\
\hline \multicolumn{2}{|l|}{ Es gibt einen standardisierten Anmeldebogen $\mathrm{a}, \mathrm{c}(90 / 92)$} \\
\hline Trifft zu & $40(44,4)$ \\
\hline \multicolumn{2}{|l|}{ Es gibt einen standardisierten Dokumentationsbogen a,c $(91 / 92)$} \\
\hline Trifft zu & $38(41,8)$ \\
\hline \multicolumn{2}{|l|}{ Es gibt einen standardisierten Rückmeldebogen ${ }^{a, c}(90 / 92)$} \\
\hline Trifft zu & $22(24,4)$ \\
\hline \multicolumn{2}{|c|}{ Die Inanspruchnahme meiner Corona-Anlaufstelle bewerte ich als zufriedenstellend a,c (91/92) } \\
\hline Trifft zu & $57(62,6)$ \\
\hline Merkmale (Anzahl verwertbare/mögliche Rückmeldungen) & Hausarztpraxen $\mathbf{n}(\%)$ \\
\hline \multicolumn{2}{|c|}{ Über die Standorte der Corona-Anlaufstellen bin ich gut informiert ${ }^{\mathrm{a}, \mathrm{c}}(62 / 63)$} \\
\hline Trifft zu & $54(87,1)$ \\
\hline \multicolumn{2}{|c|}{ Über die Öffnungszeiten der Corona-Anlaufstellen bin ich gut informiert ${ }^{\mathrm{a}, \mathrm{c}}(62 / 63)$} \\
\hline Trifft zu & $48(77,4)$ \\
\hline \multicolumn{2}{|c|}{ Über die Abläufe der Corona-Anlaufstellen bin ich gut informiert ${ }^{a, c}(62 / 63)$} \\
\hline Trifft zu & $43(69,4)$ \\
\hline
\end{tabular}

spruchnahme von Corona-Anlaufstellen, verpflichtende telefonische Anmeldung und Versorgung von PatientInnen mit leichtem Verlauf bei (vermuteter) COVID-19-Erkrankung rein telefonisch oder per Video implementiert. Die initial wahrgenommene und aus mehreren Ländern berichtete Unterversorgung von Nicht-COVID-19-PatientInnen ging bis zum Befragungszeitpunkt deutlich zurück [13]. Parallel dazu wurden Corona-Anlaufstellen als neue ambulante Versorgungsformen vorwiegend unter hausärztlicher Leitung aufgebaut. Ähnliche Ansätze in der Pandemie-Struktur im primärärztlichen Setting sind in Australien, Neuseeland, Kanada, den Niederlanden, Großbritannien und den USA jeweils mit unter- schiedlichen Gewichtungen beschrieben [13]. Der Aufwand beim Aufbau der Corona-Anlaufstellen wird v. a. bei einem Großteil der Fieberambulanzen und bei fast der Hälfte der Corona-Schwerpunktpraxen als hoch eingeschätzt. Dies deckt sich mit Ergebnissen aus Schwerpunktpraxen in Berlin [14]. Auch bei HausärztInnen in Deutschland wird die Praxisorganisation als maßgebliche Herausforderung während der COVID-19-Pandemie genannt [15].

Aus der Vielzahl der beteiligten Akteure in den Corona-Anlaufstellen lässt sich die Notwendigkeit einer Zusammenarbeit mit vielen Schnittstellen z. B. in Bezug auf Information und Kommunikation ableiten. Managementlücken taten sich in den Corona-Anlaufstellen 
- Tab. 5 Zukunft/Weiterentwicklung.

\begin{tabular}{|c|c|}
\hline Merkmale (Anzahl verwertbare/mögliche Rückmeldungen) & Corona-Anlaufstellen $\mathbf{n}$ (\%) \\
\hline \multicolumn{2}{|l|}{ Möglichkeiten zur Steigerung der Inanspruchnahme der Corona-Anlaufstelle sind meines Erachtens ${ }^{\mathrm{b}}$ (78/92) } \\
\hline Informationsangebot für HausärztInnen steigern & $53(67,9)$ \\
\hline Informationsangebot für PatientInnen steigern & $58(74,4)$ \\
\hline Informationsangebot allgemein steigern & $49(62,8)$ \\
\hline Steuerung der ambulanten PatientInnen durch entsprechend äußere Regelungen während der Pandemie & $52(66,7)$ \\
\hline Steuerung der ambulanten PatientInnen durch entsprechende Abrechnungsregelungen während der Pandemie & $34(43,6)$ \\
\hline \multicolumn{2}{|l|}{ Für die kommenden 6 Wochen halte ich den Betrieb meiner Einrichtung dauerhaft für sinnvoll ${ }^{a, c}(91 / 92)$} \\
\hline Trifft zu & $65(71,4)$ \\
\hline \multicolumn{2}{|c|}{ Für die kommenden 6 Wochen halte ich bei starkem Anstieg der COVID-Fälle den Betrieb meiner Einrichtung für sinnvoll a,c $(90 / 92)$} \\
\hline Trifft zu & $87(96,7)$ \\
\hline Merkmale (Anzahl verwertbare/mögliche Rückmeldungen) & Hausarztpraxen $\mathbf{n}(\%)$ \\
\hline \multicolumn{2}{|c|}{ Für die kommenden 6 Monate halte ich die Versorgung von PAT_COV in meiner Praxis telefonisch für sinnvolla,c,d (63/63) } \\
\hline Trifft zu & $46(73,0)$ \\
\hline \multicolumn{2}{|c|}{ Für die kommenden 6 Monate halte ich die Versorgung von PAT_COV in meiner Praxis per Videosprechstunde für sinnvolla,c,d (62/63) } \\
\hline Trifft zu & $15(24,2)$ \\
\hline \multicolumn{2}{|l|}{ Für die kommenden 6 Monate halte ich die Versorgung von PAT_COV in meinen Praxisräumen für sinnvolla,c,d (63/63) } \\
\hline Trifft zu & $33(52,4)$ \\
\hline \multicolumn{2}{|l|}{ Für die kommenden 6 Monate halte ich den Betrieb der Corona-Anlaufstellen dauerhaft für sinnvoll $\mathrm{a,c}(63 / 63)$} \\
\hline Trifft zu & $32(50,8)$ \\
\hline \multicolumn{2}{|c|}{ Für die kommenden 6 Monate halte ich den Betrieb der Corona-Anlaufstellen bei starkem Anstieg der Fälle für sinnvoll a,c $(62 / 63)$} \\
\hline Trifft zu & $51(82,3)$ \\
\hline
\end{tabular}

bei der gesteuerten Anmeldung, der standardisierten Dokumentation und der Digitalisierung auf. Schon vor Beginn der COVID-19-Pandemie wurde die Performance des deutschen Gesundheitssystems im Ländervergleich in das untere Mittelfeld mit guten Leistungen in Versorgungsprozessen und Outcomes aber Verbesserungsbedarf in Zugang und administrativer Effizienz eingeordnet [16].

Die Inanspruchnahme war nur in 62,5\% der Corona-Anlaufstellen als zufriedenstellend angegeben. Häufig genannte Möglichkeiten die Inanspruchnahme zu erhöhen, war eine Steigerung der Informiertheit der HausärztInnen und PatientInnen. Den Bedarf an verlässlichen Informationen seitens der Hausärzteschaft beschreibt auch Bergmaier [15].

Bezüglich der zukünftigen Versorgung wurde eine große Heterogenität der Haltung zur Versorgung von PatientInnen mit (vermuteter) COVID-19-Erkrankung in der eigenen Praxis und der Notwendigkeit des dauerhaften Erhalts von Corona-Anlaufstellen festgestellt. Sowohl die Patiententrennung als auch fehlendes Feedback an die Entscheidungsträger sind in der Literatur als Herausforderungen im primärärztlichen Setting beschrieben [17]. In Australien sind eine starke Kommunikation mit den Primärversorgern, Feedbackschleifen und Online-Schulungen für den primärärztlichen Sektor während der COVID-19-Pandemie implementiert worden [18].

Einigkeit bestand über die Notwendigkeit des Angebots von Corona-Anlaufstellen bei starkem Anstieg von COVID-19-Fällen. Vor dem Hintergrund der Dynamik der COVID-19-Pandemie und mit Blick auf den hohen Aufwand im Aufbau insbesondere der Fieberambulanzen könnten hierfür Vorlagen für Struktur und Workflows wie in China und Südkorea hilfreich sein $[19,20]$. Der Wunsch nach Beibehalt der rein telefonischen Behandlung inklusive Arbeitsun- fähigkeitsbescheinigung wurde auch durch Bergmaier erhoben [15], die Politik steuerte erst jüngst nach einer mehrmonatigen Pause dahingehend nach. Warum der Bedarf an Videosprechstunde nur durch ein Viertel der antwortenden HausärztInnen bekundet wird, sollte Gegenstand weiterer Erhebungen sein.

Innerhalb Europas war Deutschland in der „ersten Welle“ eines der Länder mit den wenigsten Todesfällen bezogen auf die Einwohnerzahl [21]. Die Kapazitätsgrenzen der stationären Versorgung und Beatmungsplätze wurden nicht erreicht [22]. Die Auswertungen der noch anhaltenden „zweiten Welle“ stehen aus. Der Zeitpunkt der Umsetzung eines nationalen Pandemieplans sowie das Gelingen der Mobilisierung des primärärztlichen Sektors könnten einen Einfluss auf die Mortalitätsrate haben [23]. Die beschriebenen Anpassungen im primärärztlichen Setting könnten im Sinne eines „Schutzwalls“ für Krankenhäuser einen wesentlichen Beitrag dazu geleistet haben [9].

\section{Limitationen}

Der Rücklauf kann als vergleichbar mit anderen Erhebungen in Deutschland im hausärztlichen Setting beurteilt werden. Dennoch ist aufgrund der niedrigen Anzahl die Aussagekraft eingeschränkt und nur eine deskriptive Auswertung möglich. Über Gründe des niedrigen Rücklaufs der Abstrichstellen ( $n=2$ von 16) kann nur spekuliert werden. Eine Ursache könnte eine erschwerte postalische Zustellung gewesen sein bei Einrichtung außerhalb bestehender Praxisräume. Ein Non-Response-Bias kann nicht ausgeschlossen werden. Durch die Vollerhebung bei den Corona-Anlaufstellen sowie Einbezug aller Land- und Stadtkreise in Baden-Württemberg dürften die Ergebnisse dennoch einen realistischen regionalen 
Überblick geben. Für eine Verallgemeinerung (regional und national) sollten weitere Erhebungen stattfinden.

\section{Schlussfolgerung}

Mit Improvisation und durch das Zusammenspiel mehrerer Akteure gelang in einem Kraftakt eine rasche Implementierung von Anpassungen im primärärztlichen Setting während der frühen Phase der COVID-19-Pandemie. Folgende Anregungen lassen sich aus den Ergebnissen ableiten: Auf den Aspekt Kommunikation und Austausch an den Schnittstellen der Corona-Anlaufstellen könnte ein vermehrter Fokus gelegt werden. Gesteuerte Zugangswege zu Corona-Anlaufstellen sowie vollständig digitale Erhebung und Standardisierung in der Kommunikation könnten wichtige Impulse zur geregelten Versorgung von PatientInnen mit (vermuteter) COVID19-Erkrankung sein. Gleichzeitig könnte dies der Grundstein für ein Monitoring während einer Erkrankung sein, zur Datenerhebung für Forschung im primärärztlichen Setting beitragen und Grundlagen für ein ambulantes Monitoring der Bedarfe darstellen. Feedbackschleifen könnten Gründe für heterogene Haltungen, Bedarfe und mögliche umsetzbare Lösungen erheben. Für die langfristige Pandemieplanung sollten konkrete und praxistaugliche Empfehlungen in die Pandemiepläne aufgenommen und kommuniziert werden.

\section{Finanzielle Unterstützung}

Die Studie erhielt keine finanzielle Unterstützung.

\section{Interessenkonflikt}

$A B, S M, F P K, C R, S S C$ und MW geben keine Interessenskonflikte an. NBG und SSt haben im März/April je eine Fieberambulanz aufgebaut. NBG erhielt Dienstpauschalen, SSt eine Aufwandsentschädigung für den Aufbau von der KVBW.

\section{References}

[1] Bayerisches Landesamt für Gesundheit und Lebensmittelsicherheit. Übersicht der Fallzahlen von Coronavirusinfektionen in Bayern. Im Internet: https://www.Igl.bayern.de/gesundheit/infektionsschutz| infektionskrankheiten_a_z/coronavirus/karte_coronavirus/; Stand: 31.01.2021

[2] Robert Koch Institut. COVID-19: Fallzahlen in Deutschland und weltweit. Im Internet: https://www.rki.de/DE/Content/InfAZ/N/ Neuartiges_Coronavirus/Fallzahlen.html; Stand: 31.01.2021

[3] Robert-Koch-Institut. Ergänzung zum Nationalen Pandemieplan COVID-19 - neuartige Coronaviruserkrankung (4.3.2020). Im Internet: https://www.rki.de/DE/Content/InfAZ/N/Neuartiges_Coronavirus/ Ergaenzung_Pandemieplan_Covid.html; Stand: 05.11.2020

[4] Robert Koch Institut. Nationaler Pandemieplan Teil I (02.03.2017). Im Internet: https://www.gmkonline.de/documents/pandemieplan_teili_1510042222_1585228735.pdf; Stand: 31.01.2021

[5] Sozialministerium Baden-Württemberg. Influenzapandemieplan Baden-Württemberg (02.03.2020). Im Internet: https://sozialministerium.baden-wuerttemberg.de/fileadmin/redaktion/m-sm/intern/ downloads/Downloads_Gesundheitsschutz/InfluenzapandemieplanBW.pdf; Stand: 31.01.2021
[6] Sozialgesetzbuch. §72 SGB V - Sicherstellung der vertragsärztlichen und vertragszahnärztlichen Versorgung (zuletzt geändert durch Art. 311 V v. 19.6.2020). Im Internet: https://www.sozialgesetzbuch-sgb. de/sgbv/72.html; Stand: 31.01.2021

[7] Frosch H, Knöpnadel J, Liebeskind U et al. Influenzapandemie - Risikomanagement in Arztpraxen: Eine Empfehlung der Kassenärztlichen Bundesvereinigung, der Bundesärztekammer und der BGW (08/2008). Im Internet: http://www.bundesaerztekammer.de/fileadmin/user_upload/downloads/Risikomanagement_in_Arztpraxen.pdf; Stand: 31.01.2021

[8] Robert-Koch-Institut. Optionen zur getrennten Versorgung von COVID-19 Verdachtsfällen/Fällen und anderen Patienten im ambulanten und prästationären Bereich. (06.07.2020). Im Internet: https:// www.rki.de/DE/Content/InfAZ/N/Neuartiges_Coronavirus/Getrennte_ Patientenversorgung.html?nn=13490888; Stand: 31.01 .2021

[9] Korzilius H. Ambulante Versorgung: Schutzwall für Krankenhäuser. Deutsches Ärzteblatt. 2020. Dtsch Arztebl 2020; 117: A-754. B-642

[10] Rawaf S, Allen LN, Stigler FL et al. Lessons on the COVID-19 pandemic, for and by primary care professionals worldwide. Eur J Gen Pract 2020; 26: 129-133. doi:10.1080/13814788.2020.1820479

[11] Kassenärztliche Vereinigung Baden-Württemberg. Coronakarte KVBaWue. Im Internet: http://coronakarte.kvbawue.de/; Stand: 31.01.2021

[12] STROBE Statement Checklist of items that should be included in reports of cross-sectional studies. (2007) Im Internet: https://www. strobe-statement.org/index.php?id = strobe-home; Stand: 31.01 .2021

[13] Huston P, Campbell J, Russell G et al. COVID-19 and primary care in six countries. BJGP Open 2020; 4. doi:10.3399/bjgpopen20X101128

[14] Zentralinstitut für die kassenärztliche Versorgung (Zi). Zeitbedarf und Kosten für COVID-19-Leistungen in Berliner Schwerpunktpraxen Ergebnisse für die KV Berlin (10.09.2020). Im Internet; https://www. zi.de/fileadmin/images/content/PMs/COVZ_Ergebnisse_100920.pdf; Stand: 31.01.2021

[15] Bermaier C, Schneider A, Torge M et al. Fallzahlen und Herausforderungen in südbayerischen Hausarztpraxen während der COVID-19-Pandemie. ZFA 2020; 96: 357-362. doi:10.3238/zfa.2020.0357-0362

[16] Schneider EC, Sarnak DO, Squires D et al. Mirror, Mirror 2017: International Comparison Reflects Flaws and Opportunities for Better U.S. Health Care. The Commonwealth Fund; 2017

[17] Kunin M, Engelhard D, Thomas S et al. Challenges of the Pandemic Response in Primary Care during Pre-Vaccination Period: A Qualitative Study. Isr J Health Policy Res 2015; 4: 32. doi:10.1186/s13584-015-0028-5

[18] Desborough J, Hall Dykgraaf S, de Toca L et al. Australia's national COVID-19 primary care response. Med J Aust 2020; 213: 104-106. e101. doi:10.5694/mja2.50693

[19] Jiang $\mathrm{H}$, Liu JW, Ren $\mathrm{N}$ et al. Emergency management in fever clinic during the outbreak of COVID-19: an experience from Zhuhai. Epidemiol Infect 2020; 148: e174. doi:10.1017/S0950268820001764

[20] Kwon KT, Ko JH, Shin H et al. Drive-Through Screening Center for COVID-19: a Safe and Efficient Screening System against Massive Community Outbreak. J Korean Med Sci 2020; 35: e123. doi:10.3346/ jkms.2020.35.e123

[21] Arentz C, Wild F. WIP-Analyse. Vergleich europäischer Gesundheitssysteme in der Covid-19-Pandemie. Köln: WIP - Wissenschaftliches Institut der PKV; ISBN 978-3-9821682-2-72020

[22] Karagiannidis C, Mostert C, Hentschker C et al. Case characteristics, resource use, and outcomes of 10021 patients with COVID-19 admitted to 920 German hospitals: an observational study. The Lancet Respiratory Medicine 2020; 8: 853-862. doi:10.1016/s22132600(20)30316-7

[23] Goodyear-Smith F, Kinder K, Mannie C et al. Relationship between the perceived strength of countries' primary care system and COVID-19 mortality: an international survey study. BJGP Open 2020; 4 (4). doi:10.3399/bjgpopen20X101129 